\title{
Expansion of Network Integrations: Two Scenarios, Trade Patterns, and Welfare
}

\author{
Noritsugu Nakanishi \\ Kobe University \\ Toru Kikuchi \\ Kobe University
}

\begin{abstract}
We present two different scenarios of expanding the communication networks (through which the intermediate business services are traded) and examine their consequences on trade patterns in goods and welfare of the countries. The first scenario is the "successive expansion" of a single network integration and the second one is the "parallel expansion" of plural network integrations. We show that the former can have harmful effects on the outside countries, while the latter can be "Pareto-improving" in each stage of the network expansion.
\end{abstract}

- JEL Classifications: F12, F15

- Key words: Network integration, Trade pattern, Successive expension, Parallel expansion

\section{Introduction}

The advancement of communication networks such as the Internet has been bringing about changes in the international business circumstances; it reduces costs of communication between producers and consumers geographically distant from each other and provides faster ways to distribute some products and services (in

*Corresponding address: Noritsugu Nakanishi, Graduate School of Economics, Kobe University, Rokkodai-cho 2-1, Nada-ku Kobe 657-8501, Japan, Tel/Fax: +78-803-6837, E-mail: nakanishi@econ. kobe-u.ac. jp. Toru Kikuchi, Graduate School of Economics, Kobe University, Rokkodai-cho 2-1, Nada-ku, Kobe 657-8501, Japan, Tel/Fax: +78-803-6838, E-mail: kikuchi@econ.kobe-u.ac.jp (C2005-Center for International Economics, Sejong Institution, All Rights Reserved. 
particular, the intermediate business services such as informational services, business consulting, system engineering and so forth). So, it might be quite possible that the more domestic networks are interconnected with each other, the more the worldwide production-efficiency and the welfare of the countries are enhanced. In this paper we investigate this possibility.

If two countries interconnect their domestic networks mutually and form a network integration, then each of them now becomes able to make indirect use of the resources located in the partner country. In a sense, the impacts of a network integration on the production structure of its members are similar to (virtually, the same as) those of an economic integration in which goods and factors can move freely. We present two different scenarios concerning the way how network integrations expand and examine their consequences on trade patterns in goods and welfare of the countries. ${ }^{1}$ The first scenario of the network expansion is the "successive expansion" of a single network integration, in which one country joins the only existing network integration at a time and expands the size of the existing network integration by one; in this way, the unique network integration expands one by one until all the countries are integrated into the worldwide (largest) network integration. The second scenario of the network expansion is the "parallel expansion" of plural network integrations, in which pairs of countries form new network integrations of size two, then, pairs of network integrations of size two form new network integrations of size four, then, in each stage, pairs of small network integrations of the same size form larger network integrations twice as large as the original networks; in this way, many network integrations expand simultaneously until all the countries are integrated into the worldwide network integration.

Although these two scenarios share the same final situation (i.e., the worldwide network integration), their effects on trade patterns in goods and welfare of the countries (in particular, those observed before the final situation is reached) are quite different. In the course of the successive expansion, there can occur some "inter-industry trade" in final goods, and the successive expansion can have harmful effects on the welfare of the outside countries. On the other hand, in the course of the parallel expansion, only the "intra-industry trade" in the business services that are supplied through the network can occur, and the parallel expansion can be "Pareto-improving" in each stage of the network expansion.

\footnotetext{
${ }^{1}$ Some studies have presented models incorporating the features of the communication networks. Examples include Harris (1998), MacKie-Mason and Varian (1995), Kikuchi (2002, 2003), Kikuchi and Ichikawa (2002).
} 


\section{The Model}

Consider a world economy consisting of $M$ identical countries. ${ }^{2}$ Each country is endowed with $L$ units of labor. There are two consumption goods: $X$ and $Y$. The quantity of good $X$ is denoted by $x$. Each consumer's preference over the consumption of $X$ and $Y$ is represented by an identical Cobb-Douglas utility function with share coefficients $\mu$ on $X$ and $1-\mu$ on $Y$, respectively. Both goods are sold in perfectly competitive markets. Good $Y$ is produced under constant-returnsto-scale (CRS) technology using only labor; units are chosen that one unit of labor produces one unit of $Y$.

Good $X$ is produced under CRS technology using only differentiated business services, which are produced by monopolistically competitive service firms and supplied only through communication networks. The production function of good $X$ is represented by a CES function, as in Ethier (1982) and Markusen (1989): ${ }^{3}$

$$
x=\left(\int_{0}^{1} z(i)^{(\sigma-1) / \sigma} d i\right)^{\sigma /(\sigma-1)}, \sigma>1
$$

where $n$ is the range of available intermediate business services, $z(i)$ is the quantity of the $i$ th variety of business services, and $\sigma$ is the elasticity of substitution between every pair of services. Let $q(i)$ be the price of the $i$ th variety of business services, then the unit cost function for good $X$ can be written as follows:

$$
c=\left(\int_{0}^{n} q(i)^{1-\sigma} d i\right)^{1 /(1-\sigma)} .
$$

From the profit maximization condition of the good $X$ producer, we can derive the (conditional) demand function for the $i$ th variety of business services:

$$
z(i)=\frac{q(i)^{-\sigma}}{Q} x,
$$

where $Q=\left(\int_{0}^{n} q(j)^{(1-\sigma)} d j\right)^{\sigma /(\sigma-1)}$. As can be seen easily from Eq. (3), the price elasticity of the (conditional) demand for the $i$ th variety of business services is equal to $\sigma$.

Intermediate business services are supplied by monopolistically competitive service firms. The central assumption is that both the production and the distribution of business services require communications through a country-specific

\footnotetext{
${ }^{2}$ The basic model follows that of Kikuchi (2003).

${ }^{3}$ Based on a similar setting, Anwar (2001) has considered the relationship between government spending on public infrastructure and trade patterns.
} 
network, which is provided by a natural monopolist in each country. To get on the network, each service firm has to pay a fixed fee $\alpha>0$ (in terms of labor) to the network monopolist. ${ }^{4}$ Once each service firm gets on the network, it must pay a constant marginal cost $\beta>0$ (in terms of labor) per unit of its business service supplied through the network. When a service firm produces and supplies $z$ units of service, its cost in terms of labor amounts to $\alpha+\beta z$. Given Eq. (3) and the wage rate $w$, the condition for profit maximization of a service firm becomes as follows: ${ }^{5}$

$$
q\left(1-\frac{1}{\sigma}\right)=w \beta
$$

By choosing units appropriately, we can set $\beta=(\sigma-1) / \sigma$ and, therefore, we have $q=w$. Hence, the unit cost of good $X$ [Eq. (2)] is simplified to

$$
c=n^{1 /(1-\sigma)} q=n^{1 /(1-\sigma)} w .
$$

This equation implies that the unit cost decreases as the degree of input differentiation measured by $n$ increases. Note also that as $\sigma$ becomes smaller (i.e., each service is more differentiated from others), the degree of cost reduction through an increase in the range of business services becomes larger. By substituting $q=w$ into the zero-profit condition for a (representative) service firm, we obtain the long-run output of the business service per firm:

$$
\bar{z}=\alpha \sigma
$$

Before moving to the trading equilibrium, let us consider the situation in which there is no trade in goods and business services, that is, the autarky. Because good $X$ is supplied in a competitive market, the price of $X$ denoted by $p$ must be equal to its unit cost as far as a strictly positive amount is supplied: ${ }^{6}$

$$
p=c=n^{1 /(1-\sigma)}=(\alpha \sigma)^{1 / \sigma} x^{-1 / \sigma} .
$$

\footnotetext{
${ }^{4}$ This assumption implies that (i) there are aggregate constant returns in providing communications services and (ii) the pricing of communications services is done on an average-cost basis. It may be natural to assume that this connection fee is a function of a number of factors such as the number of users, market structure, and so forth [see Kikuchi (2002)]. In this note, to make the model tractable, the assumptions on network technology are very much simplified. ${ }^{5}$ We assume the symmetric equilibrium in the sense that all the service firms set equal prices and supply
equal amounts of business services.

${ }^{6}$ Note that $q=w=1$ holds because good $Y$ is produced domestically. Further, as we assumed the symmetric equilibrium, we have $n=(x / \bar{z})^{(\sigma-1) / \sigma}$ by Eq. (3).
} 
By solving the above equation for $x$ and taking account of the resource (laborendowment) constraint, we obtain the supply function $S$ for good $X$ of a country in autarky:

$$
S(p) \equiv \begin{cases}\alpha \sigma p^{-\sigma} & \text { if } p \geq \underline{c}(1) \\ \bar{x} & \text { if } 0 \leq p \leq \underline{c}(1)\end{cases}
$$

where $\bar{x} \equiv L^{\sigma^{\prime /(\sigma-1)}}(\alpha \sigma)^{1 /(1-\sigma)}$ is the maximum amount of good $X$ that can be supplied by a country determined by the resource constraint and $\underline{c}(1) \equiv(\alpha \sigma)^{1 / \sigma}(\bar{x})^{-1 / \sigma}$ is the height of the supply curve corresponding to $\bar{x}$. The downward-sloping curve $s s^{1}$ (together with the vertical segment $s^{1} \bar{x}$ ) in Fig. 1 represents the graph of the supply function for $X$.

As we assumed the Cobb-Douglas preference, the demand function for $\operatorname{good} X$ can be written as follows:

$$
x=D(p, w L) \equiv \frac{\mu w L}{p} .
$$

If a certain positive amount of good $Y$ is produced, then the wage rate is fixed at unity (i.e., $w=1$ ); on the other hand, if good $Y$ is not produced (i.e., the country completely specializes in good $X$ ), then the wage rate changes according to changes in the relative price of good $X$. The downward-sloping curve $d d^{*}$

Figure 1. A closed economy

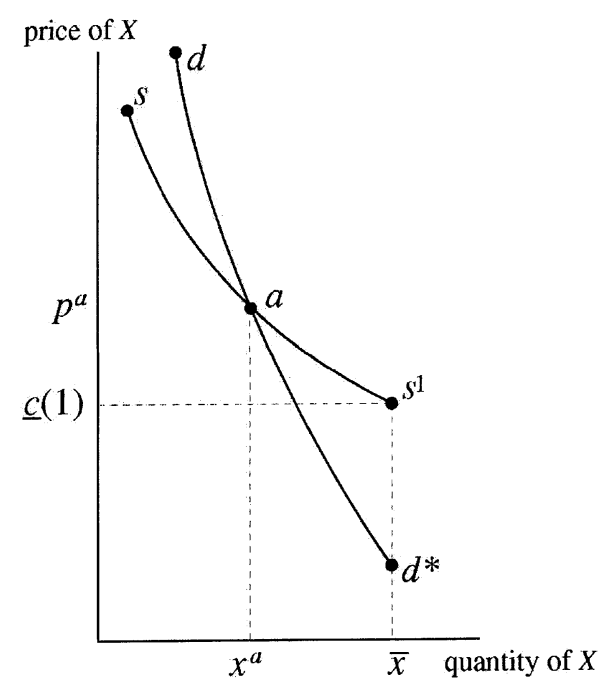


represents the graph of the demand function of a country.

Combining equations (8) and (9), we can obtain the price $p^{a}$, the quantity $x^{a}$ of $\operatorname{good} X$, and the number $n^{a}$ of the varieties of business services available in the long-run autarkic equilibrium of a country as follows:

$$
\begin{aligned}
& p^{a}=\left(\frac{\mu L}{\alpha \sigma}\right)^{1 /(1-\sigma)}, \\
& x^{a}=(\mu L)^{\sigma /(\sigma-1)}(\alpha \sigma)^{1 /(1-\sigma)}, \\
& n^{a}=\frac{\mu L}{\alpha \sigma} .
\end{aligned}
$$

In Fig. 1, the autarkic equilibrium is represented by the intersection of the supply curve $s s^{1}$ and the demand curve $d d^{*}$ (i.e., point $\left.a\right)^{7}$

\section{Two Scenarios of Network Expansion}

We consider two different scenarios of network expansion. The first scenario of network expansion is described as follows. Initially, the world economy is in a situation where the consumption goods $X$ and $Y$ are traded among the countries freely, while the business services are not traded internationally at all. From this situation, two (and only two) countries interconnect their country-specific networks and, thereby, form a network integration of size two; all the other countries do not form any other network integrations. Then, given the network integration of size two, another country joins the network integration; a new network integration of size "three" is established. In this way, one country joins the existing network integration at a time and, thereby, expands the size of the existing network integration by one. We call this scenario as the "successive expansion" of a single network integration.

The second scenario of network expansion is described as follows. Similar to the successive expansion of a single network integration, the world economy is in the situation of the goods-only-free-trade initially. From this situation, pairs of two countries interconnect their domestic networks mutually and form network integrations of size "two" simultaneously; network integrations of size two are established as many as possible. Then, pairs of network integrations of size two interconnect their networks and form new network integrations of size "four" as many as possible. Further, pairs of network integrations of size four form newer

\footnotetext{
${ }^{7}$ As can be seen from the figure, the autarkic equilibrium is unstable in the Walrasian sense, while it is
} stable in the Marshallian sense. Here, we simply assume the Marshallian stability of the equilibrium. 
network integrations of size "eight" as many as possible. In this way, two network integrations of certain size (say, size $m$ ) interconnect their country-specific networks and, thereby, form a larger network integration of size $2 \mathrm{~m}$. We call this scenario as the "parallel expansion" of plural network integrations.

\section{A. Aggregate Supply Function of a Network Integration}

Before proceeding, let us consider how the aggregate supply function for good $X$ of a single network integration changes according to its size, which is measured in terms of the number of the member countries. Now, suppose that $m$ countries have formed a network integration. Then, each member country can make use of the business services produced in other member countries through the integrated networks. This, in effect, is equivalent for each member country to become able to make use of the labor forces located in other member countries. Hence, we can treat a network integration with $m$ members (each of them possesses $L$ labor) as if it were a "single country" whose labor endowment is $m L$.

The maximum amount of good $X$ that can be supplied by a single country with $L$ units of the labor endowment is $\bar{x}$. On the other hand, the maximum amount of good $X$ that can be supplied by a network integration with $m$ members is $m^{\sigma(\sigma-1)} \bar{x}$. By Eq. (7), we can determine the height of the aggregate supply curve of a network

Figure 2. Individual supply functions and the aggregate supply function of a network integration

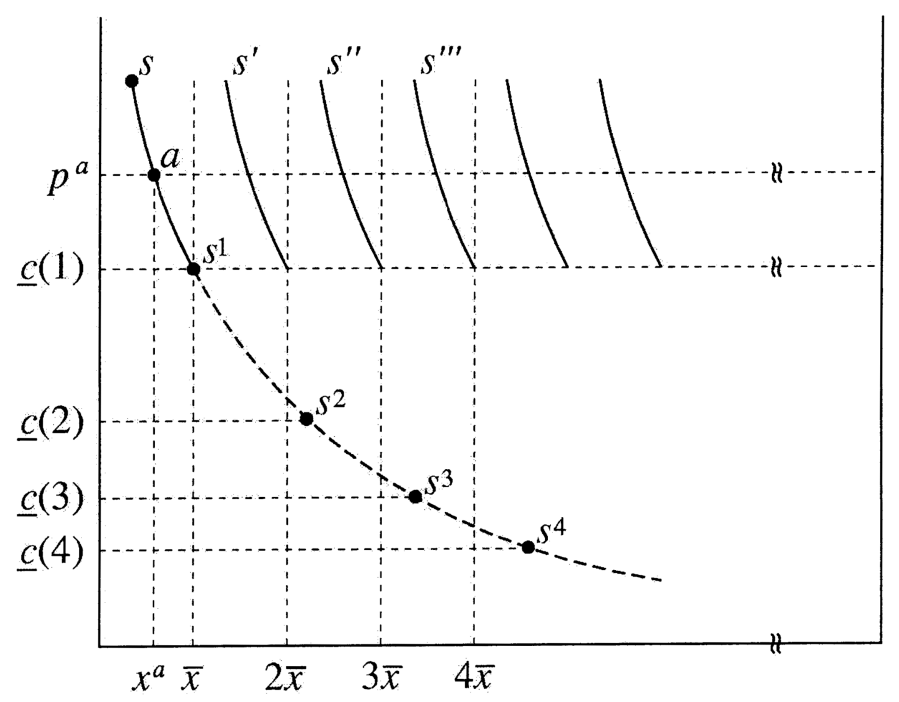


integration with $m$ members corresponding to the maximum amount $m^{\sigma(\sigma-1)} x$ :

$$
\underline{c}(m) \equiv(\alpha \sigma)^{1 / \sigma}\left[m^{\sigma /(\sigma-1)} \bar{x}\right]^{-1 / \sigma} .
$$

Clearly, we have $\underline{c}(m)>\underline{c}(m+1)$ for any integer $m \geq 1$.

Figure 2 illustrates the relationship between individual supply functions and the aggregate supply function of a network integration. As in Fig. 1, the curve $s s^{1}$ represents the supply curve of a single country. If two countries form a network integration of size two, then the aggregate supply curve is extended from $s s^{1}$ to $s s^{2}$. If another country joins the network integration of size two, then the aggregate supply curve is further extended to $s s^{3}$. The supply curve of a network integration is not a curve obtained by summing individual supply curves of the member countries horizontally, but a curve obtained by extending a single country's supply curve downward as in the figure. In sum, the supply function $S_{m}$ for good $X$ of a network integration with $m$ members can be written as follows:

$$
S_{m}(p) \equiv \begin{cases}\alpha \sigma p^{-\sigma} & \text { if } p \geq \underline{c}(m) \\ m^{\sigma /(\sigma-1) \bar{x}} & \text { if } 0 \leq p \leq \underline{c}(m)\end{cases}
$$

The above equation generalizes Eq. (8).

\section{B. Successive Expansion of a Single Network Integration}

Let us consider the first scenario of the "successive expansion" of a single network integration. Suppose that there is only one network integration consisting of $m$ countries $(1 \leq m \leq M)$. In this case, the aggregate world supply function $S_{m}^{I}$ is represented by the sum of the aggregate supply function $S_{m}$ of the network integration and $M-m$ individual supply functions of the outside countries:

$$
S_{m}^{I}(p) \equiv S_{m}(p)+(M-m) \cdot S(p) .
$$

Consider the case where there is no network integration (i.e., $m=1$ ). In this case, $S_{m}^{I}(p)$ reduces to $M \cdot S(p)$, whose graph is depicted by the curve $S_{1}^{I}$ in Fig. 3 . The curve $D D^{*}$ is the aggregate world demand curve, which can be obtained by aggregating the individual demand curves [Eq. (9)] horizontally. The free trade equilibrium with no network integration is attained at point $e^{1}$ (i.e., the intersection of the aggregate world supply curve and the aggregate world demand curve). As we have assumed the symmetry among the countries, it is easy to verify that the price of good $X$ in the free trade equilibrium with no network integration is the same as the autarkic price $p^{a}$ in each country. Consequently, no international 
Figure 3. The aggregate world supply functions and the trade equilibria

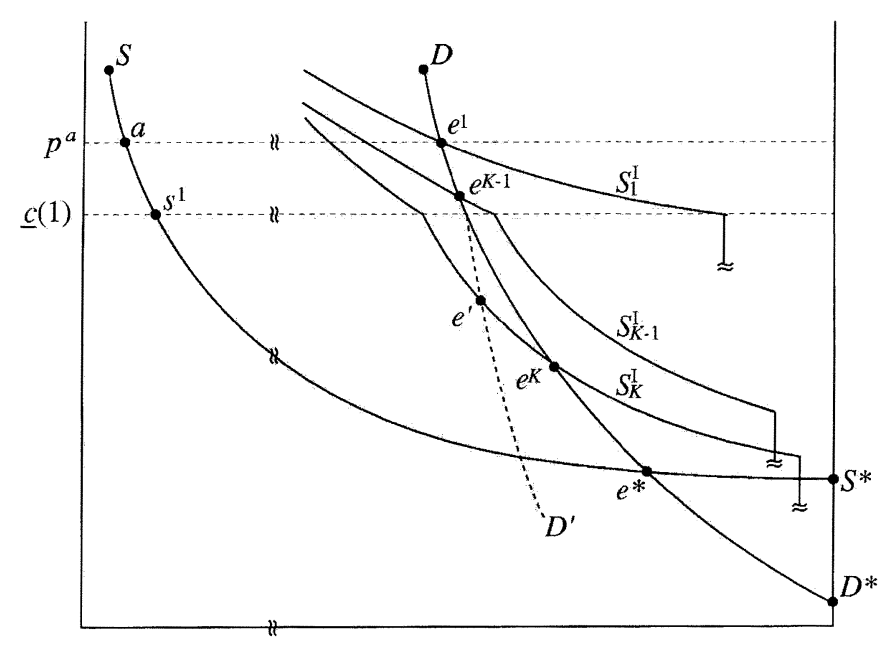

exchange of goods $X$ and $Y$ nor that of the business services takes place in the free trade equilibrium with no network integration.

Next, consider the case where all the countries form a single worldwide network integration (i.e., $m=M)$. In this case, $S_{m}^{I}(p)$ reduces to $S_{M}(p)$, whose graph is depicted by the curve $S S^{*}$ in Fig. 3. The free trade equilibrium with the worldwide network integration is attained at point $e^{*}$ (i.e., the intersection of the aggregate world supply curve $S S^{*}$ and the aggregate world demand curve $\left.D D^{*}\right)$. The equilibrium price of good $X$ becomes far lower than the autarkic price $p^{a}$. In this equilibrium, the countries exchange their differentiated business services internationally (i.e., the "intra-industry trade") on the one hand, they do not exchange the consumption goods $X$ and $Y$ on the other hand (i.e., no "inter-industry trade").

Lastly, let us consider the case where the size $m$ of the single network integration lies between 1 and $M$ (i.e., $1<m<M$ ). The aggregate world supply curve is located below $S_{1}^{I}$ and has a kink at the height of $\underline{c}(1)$ because of the existence of $(M-m)$ non-member countries; an example is the curve $S_{K}^{I}$ in Fig. 3. Further, we have $S_{m}^{I}(p)>S_{m+1}^{I}(p)$ for any $m \geq 1$ and $p \geq 0 .{ }^{8}$ That is, as the size of the single network integration increases, the aggregate world supply curve shifts downward monotonically. The larger the size of the single network integration is, the cheaper the good $X$ can be supplied. It should be noted that, for any $m$ with $1<m<M$, the

${ }^{8}$ For simplicity, we are ignoring here the vertical segments of the aggregate supply curves. 
aggregate world supply curve has a kink at the height of $\underline{c}(1)$.

Now, let us consider how the trade equilibrium changes as the size $m$ of the single network integration increases. As noted before, if there is no network integration, the equilibrium point is $e^{1}$ as in Fig. 3. This is an interior equilibrium. Hence, each country produces positive amounts of both goods $X$ and $Y$ in the equilibrium with no network integration.

As the number of the member countries of the network integration increases, the equilibrium point shifts downward and the equilibrium price falls monotonically along the aggregate world demand curve $D D^{*}$. Then, we can easily show that there exists a critical value $K$ of the number of the member countries for which the equilibrium price is below $\underline{c}(1)$, while the equilibrium price for $K-1$ members of the network integration is above $\underline{c}(1)$. The curves $S_{K-1}^{I}$ and $S_{K}^{I}$ in Fig. 3 illustrate this situation. If the size of the network integration is $K$, then we have $p<\underline{c}(1)$. In this case, each non-member country specializes in good $X$. As we have assumed the Cobb-Douglas preferences, both goods $X$ and $Y$ must be produced in strictly positive amounts in the equilibrium. Therefore, good $Y$ is produced only in the network integration. (The network integration as a whole is in the situation of the incomplete specialization.) Good $Y$ is exported from the network integration to non-member countries and, conversely, good $X$ is exported from non-member countries to the network integration; the business services are traded internationally only within the network integration.

Next, let us examine how the successive expansion affects the welfare of the countries. We distinguish three types of countries: incumbent members of the network integration, a new country who has just become a member of the network integration, and countries who stay outside of the network integration. If a new country joins the network integration while keeping the size of the network integration less than the critical value $K$, the new country as well as each incumbent member become able to use a wider range of the business services and the production costs of good $X$ for these countries are reduced. This cost reduction effect enhances the welfare of the new country and the incumbent member countries. On the other hand, the expansion of the network integration induces a lower price of good $X$ and, then, the production of good $X$ in each outside country "increases." This increase in the production of good $X$ reflects the increases in the production of the business services. Due to increasing returns in the business service sector, the real income increases and enhances the welfare of each outside country. 
If the size of the network integration exceeds the critical value $K$ by the entry of a new country, all the non-member countries come to specialize in good $X$. If the size of the network integration further increases by other countries' entry, the price of good $X$ decreases, which implies the deterioration of the "terms of trade" of each non-member country. That is, if the size of the network integration exceeds the critical value $K$, expansion of the network integration has harmful effects on the welfare of the non-member countries. Turn to the (new and incumbent) member countries, they can use a wider range of the business services and the cost reduction effect is the same as before. However, because the demand for good $X$ from the non-member countries does not increase due to the deterioration of their terms of trade, the network integration cannot increase the sales of good $X$ so much. In this case, expansion of the network integration can have harmful effects even on the welfare of the member countries.

\section{Parallel Expansion of Plural Network Integrations}

Let us consider the second scenario of the "parallel expansion" of plural network integrations. For simplicity, let us assume that the number of the countries is $2^{H}$ for some positive integer $H \geq 1$ (i.e., $M=2^{H}$ ).

In the first stage of this scenario, each country and its partner interconnect their country-specific networks and form a network integration of size two. Then, $2^{H-1}$ network integrations of size two are established. In the second stage, each network integration of size two and its partner network integration of size two interconnect their networks and form a larger network integration of size four. Then, $2^{H-2}$ network integrations of size four are established. In this way, in the $h$ th stage of the second scenario, a pair of incumbent network integrations of size $2^{h-1}$ form a new network integration of size $2^{h}$; the number of the network integrations decreases from $2^{H-h+1}$ to $2^{H-h}$. In the last $(H \mathrm{th})$ stage of the scenario, all the countries are integrated into a single worldwide network integration.

The aggregate world supply function $S_{h}^{\text {II }}$ for good $X$ in the $h$ th stage is written as follows:

$$
S_{h}^{\mathrm{II}}(p) \equiv 2^{H-h} \cdot S_{2^{h}}(p),
$$

where $S_{2^{h}}$ is obtained by substituting $m=2^{h}$ into Eq. (14). As is easily verified, we have $S_{h}^{\mathrm{II}}(p)>S_{2^{h+1}}(p)$ for any $h$ with $0 \leq h \leq H$ and any $p \geq 0 .^{9}$

\footnotetext{
${ }^{9}$ Again, we are ignoring here the vertical segments of the supply functions.
} 
The graph of the aggregate world supply function $S_{h}^{\text {II }}$ has a kink at the height of $\underline{c}\left(2^{h}\right)$. This kink corresponds to the maximum amount of good $X$ that can be supplied by the world as a whole (in other words, at this kinked point, the world as a whole specializes in the production of $\operatorname{good} X$ ); we can ignore this point, because the assumption of the Cobb-Douglas preference requires that both goods $X$ and $Y$ must be produced in strictly positive amounts in the equilibrium.

In the 0th stage of the scenario (that is, in the case with no network integration), the aggregate world supply curve and the aggregate world demand curve are the same as the case of the successive expansion (i.e., the curves $S_{1}^{I}$ and $D D^{*}$ ) and, therefore, the trade equilibrium is attained at point $e^{1}$ in Fig. 3.

As the parallel expansion of plural network integrations proceeds, the aggregate world supply curve shifts downward and the equilibrium point moves downward along the aggregate world demand curve and, accordingly, the equilibrium price of good $X$ decreases. In the last stage of the network expansion, the equilibrium point reaches point $e^{*}$ in Fig. 3.

In the course of the parallel expansion, each country belongs to a network integration of the same size. (Different countries may belong to different network integrations, but these network integrations are of the same size.) Then, in each stage of the parallel expansion, every network integration has an identical production structure and it exhibits "diversification" in goods $X$ and $Y$. Contrary to the case of the successive network expansion, there is no country who completely specializes in good $X$, in addition, there is no "inter-industry trade" in goods $X$ and $Y$ in the course of the parallel expansion. The international transactions only consist of "intra-industry trade" in the differentiated business services within each and every network integration.

Let us examine how the parallel expansion affects the welfare of the countries. In every stage of the parallel network expansion, all the countries becomes able to use a wider range of the differentiated business services and they can reduce the production costs of good $X$ and, therefore, they can attain higher real incomes. Hence, it is very much likely that the parallel expansion is "Pareto-improving."

\section{Remarks}

In models with increasing returns (as in this paper), it is well recognized that there can be multiple equilibria and that the equilibrium variables respond discontinuously to changes in the exogenous parameters. To avoid such 
complexities arising from factors other than the difference in two scenarios of network expansion, we have concentrated our attention only to the interior symmetric equilibria as far as possible. Therefore, the results obtained in this paper should not be taken as rigorous proofs of the properties nor complete characterizations of the equilibria, but they should be taken as indication of some possible consequences of two different scenarios of the network expansion. What is important is that the economic impacts of the network expansion depend not only on the final situation, but also on the way how the final situation is reached. That is, they are "path-dependent."

Received 18 June 2004, Accepted 21 December 2004

\section{References}

Anwar, S. (2001) 'Government Spending on Public Infrastructure, Prices, Production and International Trade,' Quarterly Review of Economics and Finance, 41, 19-31.

Ethier, W. J. (1982) 'National and International Returns to Scale in the Modern Theory of International Trade,' American Economic Review, 72, 389-405.

Harris, R. G. (1998) 'Internet as the GTP,' in Helpman, E. (ed.) General Purpose Technologies and Economic Growth. Cambridge: MIT Press

Kikuchi, T. (2002) 'Country-Specific Communications Networks and International Trade in a Model of Monopolistic Competition,' Japanese Economic Review, 53, 167-176

Kikuchi, T. (2003) 'Interconnectivity of Communications Networks and International Trade,' Canadian Journal of Economics, 36, 155-167.

MacKie-Mason, J. K., and Hal Varian (1995) 'Pricing Congestible Network Resources,' IEEE Journal of Selected Area in Communications, 13, 1141-1149.

Markusen, J. R. (1989) 'Trade in Producer Services and in Other Specialized Intermediate Inputs,' American Economic Review, 79, 85-95. 